\title{
After-hours esophagectomy may pose additional risk to patients with esophageal cancer
}

\author{
De-Hao Tu, Rirong Qu, Qi Wang, Xiangning Fu \\ Department of Thoracic Surgery, Tongji Hospital, Tongji Medical College, Huazhong University of Science and Technology, Wuhan, China \\ Contributions: (I) Conception and design: DH Tu, Q Wang; (II) Administrative support: X Fu, Q Wang; (III) Provision of study materials or patients: \\ X Fu, Q Wang; (IV) Collection and assembly of data: DH Tu, R Qu; (V) Data analysis and interpretation: DH Tu, R Qu; (VI) Manuscript writing: \\ All authors; (VII) Final approval of manuscript: All authors. \\ Correspondence to: Qi Wang. Department of Thoracic Surgery, Tongji Hospital, Tongji Medical College, Huazhong University of Science and \\ Technology, 1095 Jie Fang Avenue, Wuhan 430030, China. Email: wangqidoc@163.com; Xiangning Fu. Department of Thoracic Surgery, Tongji \\ Hospital, Tongji Medical College, Huazhong University of Science and Technology, 1095 Jie Fang Avenue, Wuhan 430030, China. Email: \\ fuxn2006@aliyun.com.
}

Background: The increase in the incidence of esophageal cancers (ECs) combined with fewer surgeons working at large centers will increase the likelihood of surgery for ECs being performed during later hours. This study aimed to compare esophagectomies' operative outcomes for EC performed at different surgical starting times.

Methods: This was a single-center, retrospective study. Risk-adjusted cumulative sum curve analysis and Cox regression analysis were used to identify the potential change-point of surgical starting times. The participants were then divided into 2 groups according to the change-point time. Propensity score matching was used to control confounding factors between the 2 groups. We compared the short- and long-term outcomes in both groups.

Results: A total of 702 patients who underwent potentially radical esophagectomy from 7 May 2014 to 31 December 2017 in our institute were included. The 3-year all-cause mortality showed a significant changepoint at $16: 42$, with an increment from $56.5 \%$ to $76.9 \%(\mathrm{P}=0.043)$. Esophagectomy that commenced between 17:00-18:59 was associated with significantly lower overall survival (OS) [multivariate hazard ratio (HR): $2.47 ; 95 \%$ confidence interval (CI): 1.25 to 4.90; $\mathrm{P}=0.010]$ and disease-free survival (DFS) (multivariate HR: 2.14; 95\% CI: 1.08 to $4.21 ; \mathrm{P}=0.028$ ). The participants were allocated to the during-hours group and the after-hours group according to the change-point of 17:00. A total of 84 participants in the during-hours group were matched to 33 participants in the after-hours group. The median operative time was shorter in the after-hours group $[309$ (during-hours) vs. 239 (after-hours) minutes, $\mathrm{P}=0.014$ ); the after-hours group had a greater incidence of respiratory complications $(22.63 \%$ vs. $45.45 \%, \mathrm{P}=0.023)$ and 90 -day mortality $(0$ vs. $9.09 \%, \mathrm{P}=0.021)$. The 5-year OS $(\mathrm{P}=0.042)$ and $\mathrm{DFS}(\mathrm{P}=0.030)$ were significantly higher in the during-hours group.

Conclusions: Esophagectomies started during after-hours are correlated with poorer surgical outcomes. It is recommended to cancel selective esophagectomies due to commence after 17:00.

Keywords: Esophageal neoplasms; esophagectomy; surgical starting time; propensity score-matched; prognosis

Submitted Oct 24, 2020. Accepted for publication Dec 24, 2020.

doi: $10.21037 /$ jtd-20-3141

View this article at: http://dx.doi.org/10.21037/jtd-20-3141 


\section{Introduction}

Esophagectomy is an important part of the radical treatment of esophageal cancer (EC) (1-3). However, performing esophagectomy demands very high skills, takes a long time, and is accompanied by a high chance of morbidity and mortality than most other surgical procedures (4-6). It has been repeatedly noticed that surgical expertise is closely related to the short- and long-term prognosis of EC (7-10), emphasizing the value of surgeons' surgical techniques and performance in the treatment of EC. Meanwhile, these findings support the centralization of EC surgeries, with fewer surgeons working at large centers $(8,9)$, which will inevitably increase the likelihood of operations being conducted during after-hours. Long hours of work may lead to inattention, lack of sleep, and fatigue of surgeons, which may impact surgery outcomes (11).

Many previous studies have shown that the operation start-time has a significant impact on patients' prognosis in general, including vascular surgery, cardiac surgery, and neurosurgery (12-17). A large multicenter study from Sweden showed that patients who underwent EC surgery on Monday and Tuesday had a higher 5-year survival rate than those who underwent the same procedure from Wednesday to Friday (18), further suggesting that the timing of surgery may affect the outcome of esophageal surgery.

To the best of our knowledge, no previous study has evaluated the relationship between the start time of the day and the outcome of the operation. We presumed that esophagectomy's surgical outcomes would worsen if the operation were started at a late time of the day. The purpose of this study was to compare the surgical and survival outcomes of patients undergoing potentially radical esophagectomy performed at different surgical starting times. We present the following article in accordance with the STROBE reporting checklist (available at http://dx.doi. org/10.21037/jtd-20-3141).

\section{Methods}

\section{Participant selection}

We investigated patients who underwent potentially radical esophagectomy between 7 May 2014 to 31 December 2017 in our institute. The exclusion criteria included pathology other than squamous cell carcinoma or adenocarcinoma, multiple primary tumors, recurrent cases, and palliative surgery, considering that neoadjuvant therapy was used mainly in more recent years, and the surgeries were conducted mostly in the morning, patients with preoperative neoadjuvant therapy were also excluded. The patient recruitment process is shown in Figure 1. The main outcome measures were operative time, estimated blood loss, complications, postoperative hospital stay, lymph node dissection, positive resection margin, and survival outcome. The patient's age, gender, body mass index, American Association of Anesthesiologists (ASA) score, history of smoking and drinking, medical comorbidities, tumor location, tumor node metastasis (TNM) staging, and mode of operation were retrospectively collected from electronic medical records. According to the well-validated Charlson comorbidity index (CCI) scoring system (19), comorbidity was assessed. The study was conducted in accordance with the Declaration of Helsinki (as revised in 2013). The study was approved by the institutional review board of Tongji Hospital, Tongji Medical College, Huazhong University of Science and Technology (NO.: TJ-C20201203) and individual consent for this retrospective analysis was waived.

\section{Surgeon validation}

All operations were performed by 5 qualified thoracic surgical teams and 18 surgeons in our institution.

\section{Surgical procedure}

The McKeown approach was performed for participants who had upper thoracic EC to ensure the resections were margin-free. The operation of middle and lower thoracic EC was mainly based on the preoperative evaluation.

In the McKeown approach, thoracotomy or videoassisted thoracoscopic surgery (VATS) is performed to mobilize esophageal and resect mediastinal lymph nodes. Then, a tubular stomach was created and anastomosed by a disposable circular stapler at the left neck. Upper abdominal and mediastinal lymph nodes, including those along the bilateral recurrent laryngeal nerve, were routinely radically resected. Cervical lymphadenectomy was be performed only when cervical lymph node metastases were suspected upon ultrasound or computed tomography (CT) scan.

In the Ivor-Lewis approach, tubular gastroplasty and abdominal lymph node dissection were performed through laparoscopic or epigastric median incision first, and then the esophagus was dissociated and resected through the fifth posterolateral intercostal incision or VATS. Finally, a disposable circular stapler was used for anastomosis at the top of the thoracic cavity. The extent of lymph node 


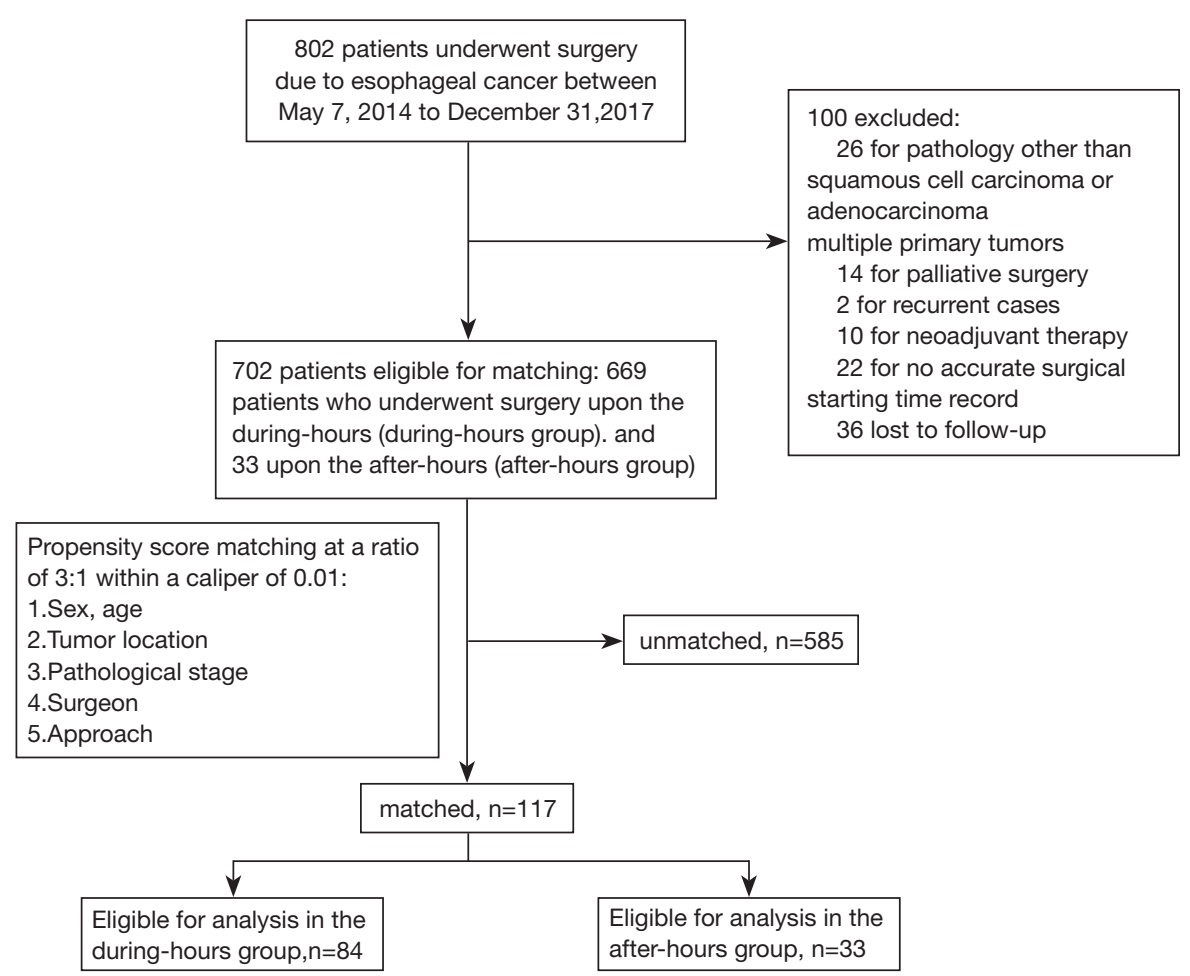

Figure 1 Flowchart of participant selection.

dissection was the same as in the McKeown approach.

In the Sweet approach, left posterolateral thoracotomy was performed through an incision in the sixth or seventh intercostal. After complete mobilization of the esophagus, the diaphragm was incised to expose the abdominal cavity and create a tubular stomach. The anastomosis was performed above or below the aortic arch. The lower mediastinum and abdominal lymph nodes were routinely resected.

Minimally invasive esophagectomy (MIE) was defined when both the thoracic and abdominal parts of the surgery are carried out using endoscopy.

All participants received enteral nutritional support through a duodenal nutritional tube or jejunostomy tube. According to the eighth edition of the Union for International Cancer Control and the American Joint Committee on Cancer (UICC/AJCC) TNM classification (20), the pathological tumor stages were evaluated. Lymph node stations were named according to the Japanese Classification for Esophageal Cancer (21).

\section{Postoperative management}

The patient was admitted to the intensive care unit (ICU) immediately after recovery from anesthesia. The nutritional tube was removed when the diet had satisfactorily returned. According to the Clavien-Dindo system $(22,23)$, postoperative complications are defined as grade 2 or above complications. Postoperative pulmonary complications were pneumonia, respiratory failure, or acute respiratory distress syndrome (ARDS). Cardiovascular complications, such as arrhythmia and heart failure, were recorded. The clinical anastomotic fistula was diagnosed in obvious clinical features or confirmed by esophagography, endoscopy, or methylene blue test. Other complications, including hoarseness and chylothorax, were also recorded. Postoperative death was defined as death within 90 days of operation (24).

\section{Follow-up}

A telephone follow-up was generally carried out every 3 months for the first 2 years and 6 months for the next 3 years. The medical reports would be requested from the patient if tumor recurrence was suspected. The overall survival (OS) time was measured in months from the date of surgery to death or last follow-up. Disease-free survival (DFS) was defined as the time from surgery to recurrence 
or death from any cause, and participants with a positive resection margin were excluded from the DFS calculation.

\section{Statistical analysis}

The statistical analyses were performed using the STATA 16.0 software (Stata Corp., College Station, TX, USA). Data were presented as number (n) and percentage (\%) for categorical data or median and interquartile range (IQR) for continuous variables. All statistical testing was 2 -sided, and $\mathrm{P}$ values $<0.05$ were considered statistically significant.

\section{Risk-adjusted cumulative sum curve analysis}

We created a risk-adjusted cumulative sum (RA-CUSUM) curves to define the performance-time curve for 1-, 2-, and 3-year all-cause mortality after esophagectomy (25). Using logistic regression, risk prediction models were employed to calculate the predicted probability of each outcome in each case (the expected mortality/survival). Potential confounding factors included in the models were age (continuous variable), gender (male or female), nutritional status (normal, underweight, or overweight), smoking index $(0,0-1,000$, or $>1,000)$, alcohol addiction (yes or no), CCI (0, 1, or $>1)$, ASA (I, II, or III), tumor pathology (adenocarcinoma or squamous cell carcinoma), pathologic stage (stage 0-I, II, III, or IV), tumor location (upper, middle, or lower), approach (Sweet, Ivor-Lewis, or McKeown), MIE (yes or no), and surgeon with the high professional title (yes or no). The curves plotted the cumulative difference between the observed and expected mortality according to the risk-adjustment model. This was calculated using the CUSUM equation $\mathrm{Si}=\mathrm{Si}-1+\left(\sum \mathrm{i}-\sum \mathrm{R}\right) ; \mathrm{S} 0=0: \mathrm{Si}$ is the cumulative sum, $\Sigma \mathrm{i}$ the sum of events at surgical starting time $i$, and $\sum R$ the sum of expected events at surgical starting time i. On the basis of this equation, the curve increased if the observed mortality/survival exceeded the expected mortality/ survival and vice versa. Change-points were identified as the maximal deflection of the curve from 0 . The changepoints' statistical significance was analyzed by comparing mortality before and after the change-points, using Fisher's exact test.

\section{Cox regression analysis}

Surgical starting times were then analyzed concerning the survival outcomes, using a multivariable Cox-proportional hazards model, and providing hazard ratios (HRs) with 95\% confidence intervals (CIs). The HRs were adjusted for all 13 potential confounding factors listed above (with the same categorization).

\section{Propensity score-matched analysis}

Based on the above results, we divided the participants into 2 groups: those operated upon within during-hours (before 17:00) and those upon within the after-hours (after 17:00). Selected cases from the 2 groups were matched at a ratio of $3: 1$ using the nearest-neighbor method with a caliper width of 0.01 . The propensity scores were developed from a logistic regression model with covariates consisting of gender, age, tumor location, pathological stage, surgeon with senior professional title, and approach. The Wilcoxon rank-sum test was applied for comparing continuous variables, while for categorical data, Fisher's exact test was applied to compare variables between the during- and afterhours groups in both the unmatched and matched cohorts. The OS and DFS were analyzed with the Kaplan-Meier method, and the log-rank test evaluated between-group differences.

\section{Results}

A total of 702 patients who underwent radical esophagectomy between 7 May 2014 and 31 December 2017 in our institute were included in this study. Complete descriptions of participant and tumor characteristics are listed in Table 1.

\section{Surgical starting time and change-points in mortality}

The RA-CUSUM analyses are illustrated in Figure 2. The pivotal change-point for 1-year all-cause mortality was 15:34, with an increment from $16.9 \%$ to $23.8 \%(\mathrm{P}=0.098$; Figure $2 A)$. The mortality rate was comparable before and after the change-point of 13:22 of 2-year all-cause mortality ( $38.15 \%$ vs. $36.89 \%, \mathrm{P}=0.806$; Figure $2 B$ ). The RA-CUSUM analysis of 3-year all-cause mortality showed that $16: 42$ was a significant change-point, with an increment from $56.5 \%$ to $76.9 \%(\mathrm{P}=0.043$; Figure $2 C$ ).

A multivariate Cox proportional hazards model also showed the different survival outcomes of various surgical starting times (Table 2). Surgery that was started between 17:00 and 18:59 was associated with significantly lower OS (multivariate HR: 2.47; 95\% CI: 1.25 to 4.90; 
Table 1 Characteristics before and after propensity score matching

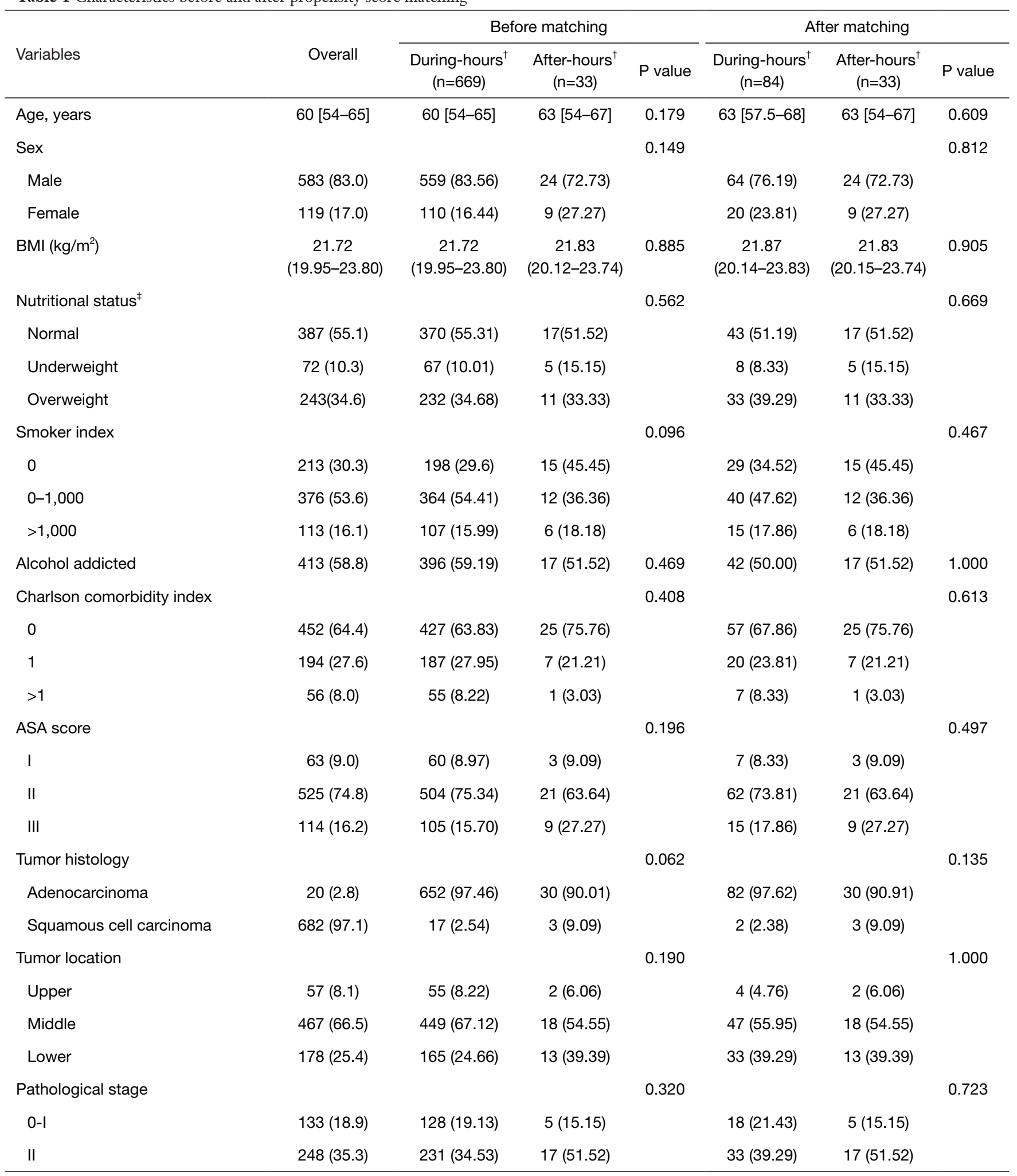

Table 1 (continued) 
Table 1 (continued)

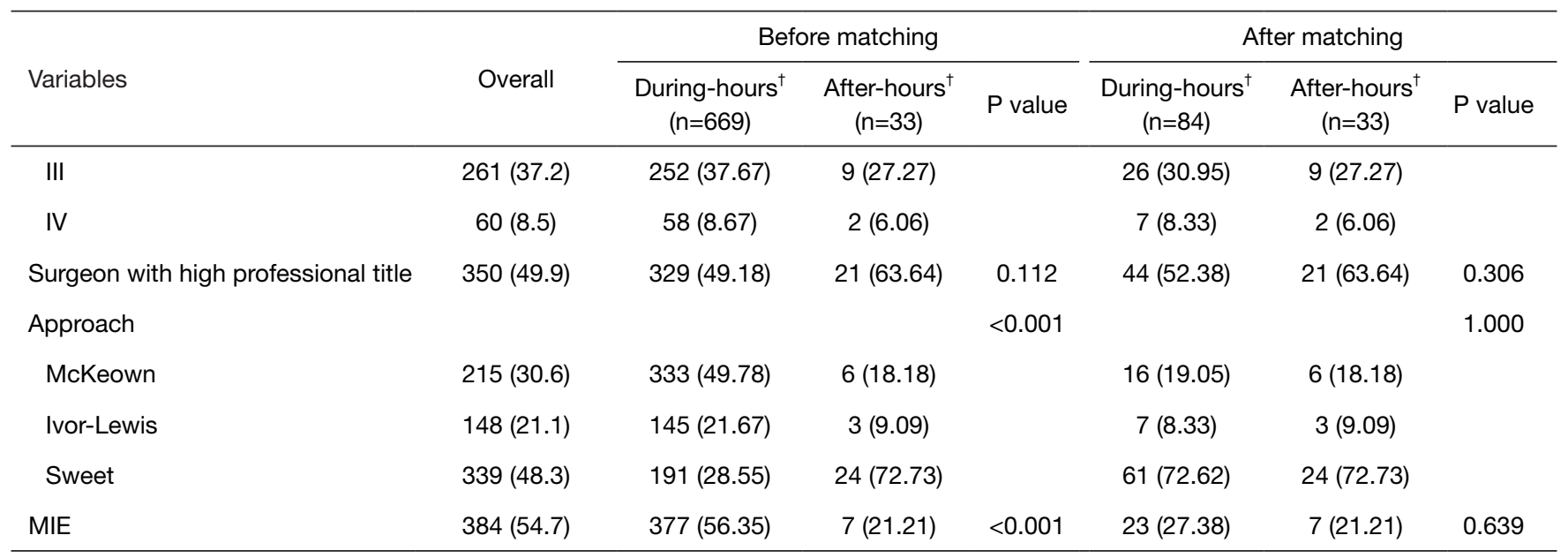

Values are given as median (IQR) or $\mathrm{n}(\%) .{ }^{\dagger}$, during-hours group: participants who underwent surgery that started before 17:00; after-hours group: participants who underwent surgery that started after 17:00; ${ }^{\ddagger}$, nutritional status defined by Asian adult standard, which is underweight, BMI below 18.5; and overweight, BMI over 23.0. MIE, minimally invasive esophagectomy.

$\mathrm{P}=0.010$ ) and DFS (multivariate HR: 2.14; 95\% CI: 1.08 to 4.21; $\mathrm{P}=0.028)$.

\section{Characteristics before and after propensity score matching}

Before propensity score matching, the after-hours group had a significantly larger proportion of the Sweet approach and a lower rate of MIE than the during-hours group $(\mathrm{P}<0.001$, Table 1). After matching, the 2 groups (84 in the during-hours group and 33 in the after-hours group) did not differ significantly in any participant characteristics (Figure 1; Table 1).

\section{Peri-operative outcomes}

Peri-operative outcomes are summarized in Table 3. The median operative time was shorter in the after-hours than the during-hours group before and after matching $(\mathrm{P}=0.001$, 0.014 , respectively). The after-hours group had a greater incidence of respiratory complications than the duringhours group both before and after matching $(\mathrm{P}=0.026,0.023$, respectively). The incidence of cardiac complications was higher in the after-hours group before matching $(\mathrm{P}=0.035)$. The after-hours group had 3 cases of 90-day mortality (1 case of respiratory and heart failure and 2 cases of sepsis caused by intrathoracic anastomosis leak), while the duringhours group had none after matching. The median bleeding volume, duration of postoperative hospitalization, and other complications did not significantly differ between the during- and after-hours groups either before or after matching.

\section{Pathologic and oncologic outcomes}

The pathologic and oncologic outcomes are summarized in Table 4. There were no significant differences between the 2 groups regarding the median numbers of harvested lymph nodes, the incidence of positive resection margin, TNM stage, depth of invasion, $\mathrm{N}$ - classification, or degree of differentiation either before or after matching.

\section{Survival outcomes}

The median time of follow-up for the whole cohorts was 25 months (IQR,14-36 months), in which the median follow-up in the during-hours group was 25 months (IQR, 14-37 months) and 24 months (IQR, 10-30 months) in the after-hours group. The 5-year OS rates did not significantly differ between the 2 groups before matching $(\mathrm{P}=0.067)$, but were significantly different after matching $(\mathrm{P}=0.042)$ (Figure 3). The DFS was significantly longer in the duringhours group both before and after matching $(\mathrm{P}=0.033$, 0.030) (Figure 3).

\section{Discussion}

There are limitations to medical resources worldwide, and this is especially so in China. Many surgeons have 
A

1-Year All-Cause Mortality

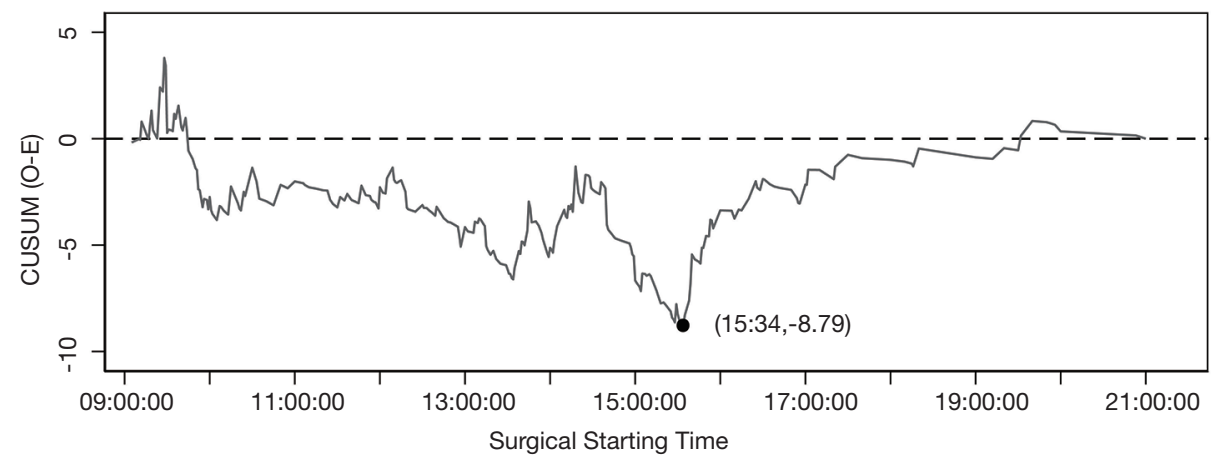

B

2-Year All-Cause Mortality

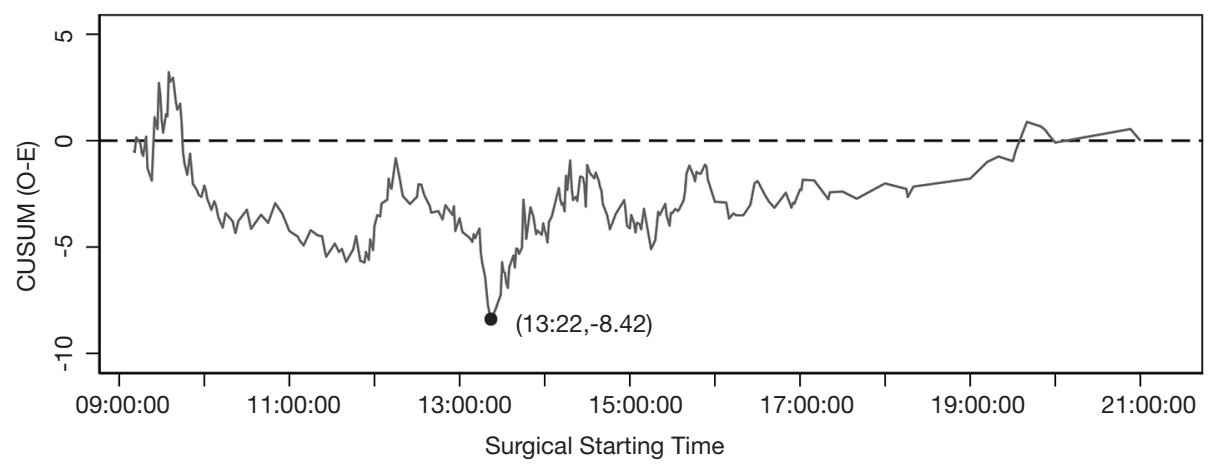

C

3-Year All-Cause Mortality

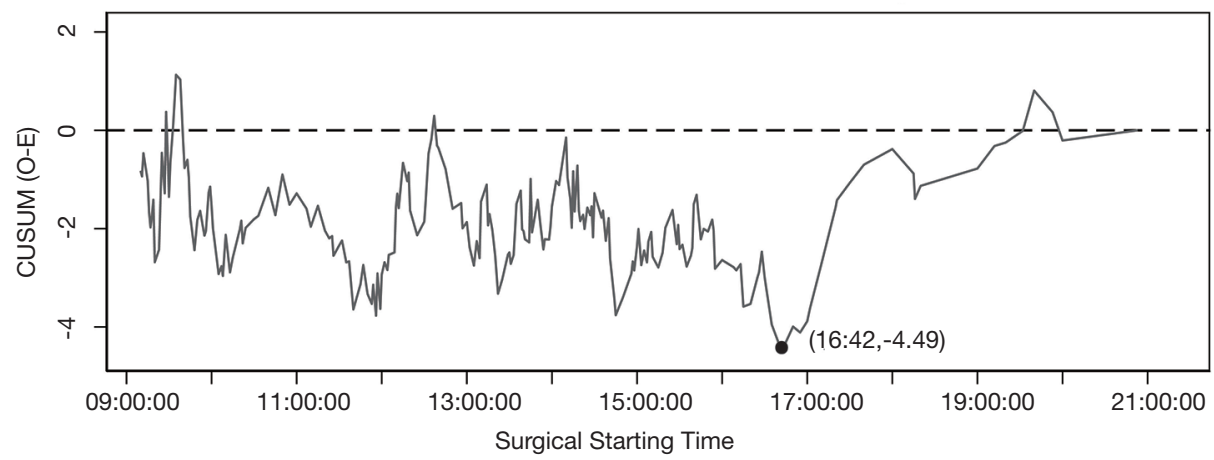

Figure 2 RA-CUSUM curve for 1- (A), 2- (B), and 3-year (C) all-cause mortality, showing change-points at 15:34 ( $\mathrm{P}=0.098), 13: 22(\mathrm{P}=0.806)$, and 16:42 ( $\mathrm{P}=0.043)$. RA-CUSUM, risk adjusted cumulative sum; $\mathrm{E}$, expected; $\mathrm{O}$, observed.

performed operations both day and night, and selective operations can begin at various time points, including late night or even early morning, which might not fully support the doctors' well-being. This article showed that potentially radical esophagectomy performed during the after-hours was associated with increased pulmonary complications and poorer long-term outcomes of EC patients.

The influence of start time on surgical results has been widely studied except in the case of surgery on the esophagus. Kelz et al. (14) detected a $25 \%$ increased risk of death for operations starting between 16:00 and 18:00 compared with those started between 07:00 and 16:00, and Linzey et al. (26) reported that compared with starting earlier in the day, the risk of developing complications was significantly higher if a neurological surgery was started between 21:01 and 07:00. However, neither of the studies made a detailed distinction among specific 
Table 2 Cox proportional hazards model of participants who underwent esophagectomy

\begin{tabular}{|c|c|c|c|c|c|}
\hline Start time & Number (\%) & Crude HR $(95 \% \mathrm{Cl})$ & Crude $P$ value & Adjusted $\mathrm{HR}^{\dagger}(95 \% \mathrm{Cl})$ & Adjusted $\mathrm{P}^{\dagger}$ value \\
\hline 9:00-10:59 & $217(30.9)$ & 1 (reference) & 1 (reference) & 1 (reference) & 1 (reference) \\
\hline $11: 00-12: 59$ & $95(13.5)$ & $0.84(0.58-1.21)$ & 0.339 & $1.06(0.69-1.63)$ & 0.801 \\
\hline $13: 00-14: 59$ & $232(33.0)$ & $0.71(0.53-0.95)$ & 0.022 & $0.96(0.66-1.41)$ & 0.854 \\
\hline 17:00-18:59 & $18(2.6)$ & $1.39(0.75-2.60)$ & 0.297 & $2.47(1.25-4.90)$ & 0.010 \\
\hline 19:00- & $15(2.1)$ & $1.18(0.55-2.53)$ & 0.679 & $1.49(0.65-3.43)$ & 0.348 \\
\hline \multicolumn{6}{|c|}{ Disease-free survival } \\
\hline 9:00-10:59 & 217 (30.9) & 1 (reference) & 1 (reference) & 1 (reference) & 1 (reference) \\
\hline $15: 00-16: 59$ & $125(17.8)$ & $0.84(0.61-1.15)$ & 0.272 & $1.16(0.77-1.74)$ & 0.478 \\
\hline $17: 00-18: 59$ & $18(2.6)$ & $1.28(0.69-2.39)$ & 0.428 & $2.14(1.08-4.21)$ & 0.028 \\
\hline 19:00- & $15(2.1)$ & $1.24(0.60-2.53)$ & 0.559 & $1.89(0.87-4.14)$ & 0.110 \\
\hline
\end{tabular}

${ }^{\dagger}$, adjustment was performed for age, gender, nutritional status, smoking index, alcohol consumption, Charlson comorbidity index, ASA, tumor pathology, pathologic stage, tumor location, approach, MIE, and surgeon with high professional title. HR, hazard ratio; Cl, confidence interval.

Table 3 Peri-operative outcomes

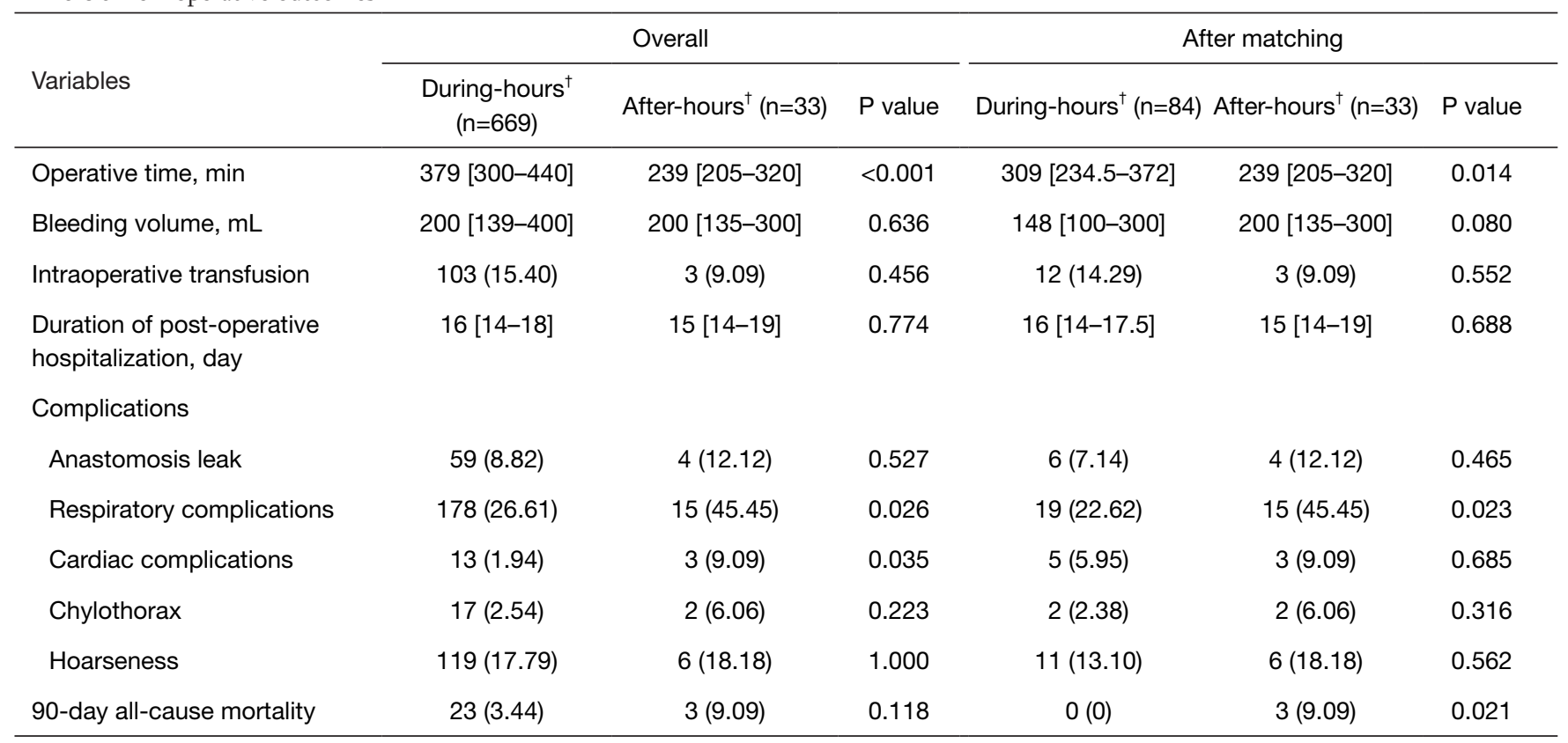

Values are given as median [IQR] or $\mathrm{n}(\%) .{ }^{\dagger}$, during-hours group: participants who underwent surgery that started before 17:00, afterhours group: participants who underwent surgery that started after 17:00. 
Table 4 Post-operative pathological outcomes

\begin{tabular}{|c|c|c|c|c|c|c|}
\hline Variables & \multicolumn{3}{|c|}{ Before matching } & \multicolumn{3}{|c|}{ After matching } \\
\hline Depth of tumor & & & 0.735 & & & 0.303 \\
\hline Tis-T1 & $111(16.59)$ & $3(9.09)$ & & $15(17.86)$ & 3 (9.09) & \\
\hline $\mathrm{T} 2$ & $163(24.36)$ & $9(27.27)$ & & $24(28.57)$ & $9(27.27)$ & \\
\hline $\mathrm{T} 4$ & $57(8.52)$ & $3(9.09)$ & & $2(2.38)$ & 3 (9.09) & \\
\hline $\mathrm{N}$ classification & & & 0.564 & & & 0.836 \\
\hline No & $375(56.05)$ & $23(69.70)$ & & $52(61.90)$ & $23(69.70)$ & \\
\hline N1 & $173(25.86)$ & $6(18.18)$ & & $15(17.86)$ & $6(18.18)$ & \\
\hline Degree of differentiation & & & 0.294 & & & 0.725 \\
\hline G1 & $109(16.95)$ & $7(21.21)$ & & $17(21.25)$ & $7(21.21)$ & \\
\hline G2 & $337(52.41)$ & $20(60.61)$ & & $43(53.75)$ & $20(60.61)$ & \\
\hline G3 & $197(30.64)$ & $6(18.18)$ & & $20(25.00)$ & $6(18.18)$ & \\
\hline Positive resection margin & $22(3.29)$ & $1(3.03)$ & 1.000 & $0(0.00)$ & $1(3.03)$ & 0.282 \\
\hline Number of the metastatic lymph node & $0[0-2]$ & $0[0-1]$ & 0.284 & $0[0-2]$ & $0[0-1]$ & 0.486 \\
\hline Number of the harvested lymph node & 16 [11-23] & 20 [14-26] & 0.198 & 18 [11.5-23] & 20 [14-26] & 0.444 \\
\hline $\begin{array}{l}\text { Number of stations of the metastatic } \\
\text { lymph node }\end{array}$ & $0[0-1]$ & $0[0-1]$ & 0.314 & $0[0-1]$ & $0[0-1]$ & 0.621 \\
\hline
\end{tabular}

Values are given as median [IQR] or $\mathrm{n}(\%) .^{\dagger}$, during-hours group patients who underwent surgery that started before 17:00, after-hours group patients who underwent surgery that started after 17:00; ${ }^{\ddagger}$, lymph node stations were named according to Japanese classification (21).

diseases or operations, so the difference might somehow be attributed to more urgent situations presenting at night. As for thoracic organ transplants, surgical outcomes were comparable between day and night (27). However, because transplant teams have developed more sophisticated systems to cope with various situations during the nighttime (27), such attribution may not apply to cancer surgery.

Logically and empirically, surgery for a severe case is not likely to be scheduled during late hours, which contradicts the worse prognosis of surgery at night. Furthermore, both Cox regression analysis and propensity score matching analysis showed that after adjusting for confounding factors, after-hours surgery's harm became even more prominent. Besides, the after-hours intervention's surgery time was shorter, which also indicated that the after-hours group contained a comparable severity of participants, if not even less complicated.

However, a possible explanation of short operative times during the after-hours would be that the later hours engendered hasty and rough work. This phenomenon was mirrored in the work of Kelz et al. (14) and Linzey et al. (26). Surgical manipulation alone is one of the causes of pulmonary damage (28), which might be more severe with hasty and rough work. This is a potential explanation for the increased pulmonary complications during the afterhours. Although the ICU and anesthesiology department was addressing the same disease manifestations during the different shifts of during- and after-hours in our 
A

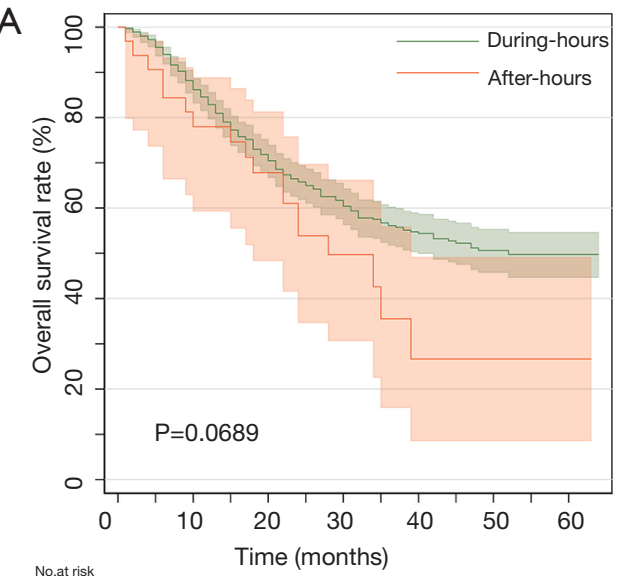

$\begin{array}{llllllllllllll}\text { During-hours } & 653 & 628 & 562 & 492 & 423 & 338 & 283 & 210 & 155 & 104 & 60 & 44 & 30\end{array}$

$\begin{array}{llllllllllllll}\text { After-hours } & 32 & 29 & 25 & 23 & 20 & 14 & 10 & 6 & 3 & 2 & 2 & 1 & 1\end{array}$

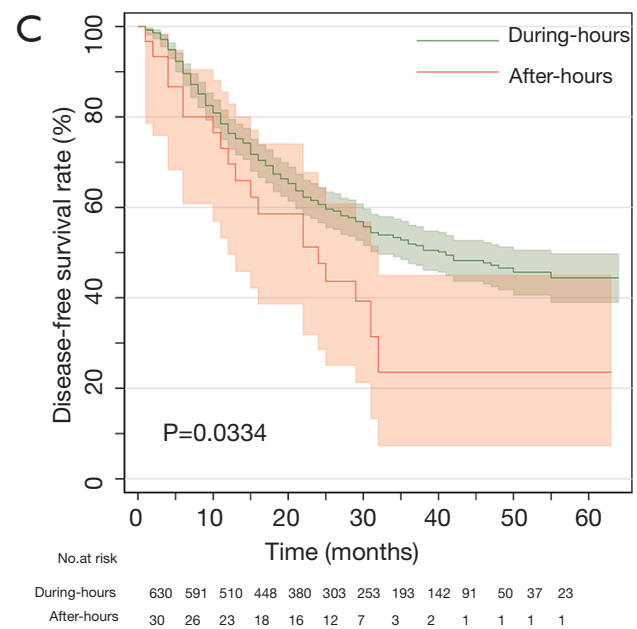

B

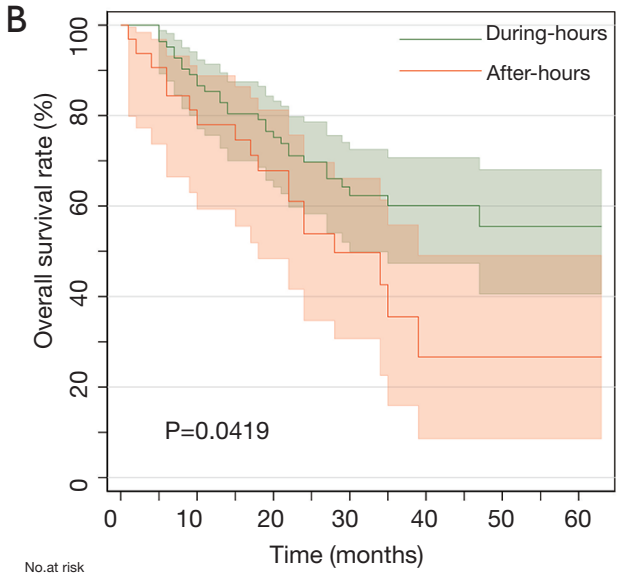

$\begin{array}{llllllllllllll}\text { During-hours } & 84 & 83 & 72 & 65 & 57 & 44 & 34 & 28 & 22 & 13 & 9 & 7 & 4\end{array}$

$\begin{array}{lllllllllllllll}\text { After-hours } & 32 & 29 & 25 & 23 & 20 & 14 & 10 & 6 & 3 & 2 & 2 & 1 & 1\end{array}$

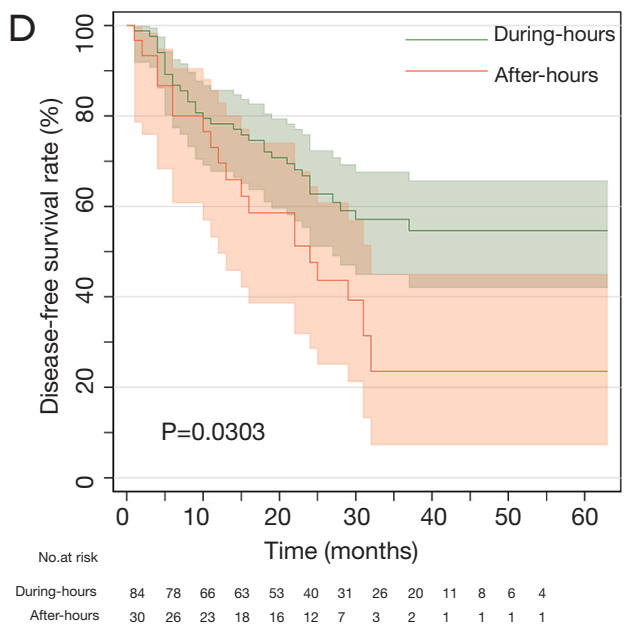

Figure 3 Kaplan-Meier curves for 5-year OS with 95\% CIs among all patients (A), and after propensity score matching (B), and that for 5-year DFS with 95\% CI among all patients (C), and after propensity score matching (D). Differences between the groups were assessed by the log-rank test. During-hours group: participants who underwent surgery that started before 17:00; after-hours group: patients who underwent surgery that started after 17:00. OS, overall survival; CI, confidence interval; DFS, disease-free survival.

institution, their judgment and performance may decrease during night hours, leading to relatively poorer respiratory management. Higher rates of open surgery may also lead to increased pulmonary complications. Furthermore, postoperative pulmonary complications were considered to be an independent predictor of poorer long-term survival in patients undergoing resection of esophageal squamous cell carcinomas (29). Since the lymph nodes harvested and the rate of positive resection margin was not significantly different between the 2 groups, the worse long-term prognosis might also be attributed to the likelihood of deviation from no-touch principles when surgeons were rushing to complete the operation.
Still, there are other reasons for the worse results of after-hours surgery. Firstly, prolonged working hours would lead to a decline in the level of performance. Research from Germany has shown that the risk of accidents increased exponentially after the 9th hour of work (30). Also, nocturnal surgery might be subject to intraoperative shift changes (31) and changes in nighttime staffing patterns (32). Anesthetic adverse events were also more likely to occur at the end of the workday (33).

The current research had some limitations. Firstly, this was a retrospective single-center study. Therefore, the results were unavoidably affected by the specific work system and environment. Also, there was a very small 
number of surgeries included that were performed during the after-hours. Subgroup analysis could not be conducted according to different surgeons, stages, or the 3 different surgical approaches. Although we tried to reduce selection bias using various methods, it was impossible to eliminate subjective judgment on treatment determination.

Additionally, it was difficult to draw a reliable conclusion about how the after-hours engendered a worse prognosis since a wide variety of factors may play roles, and our study only contained a limited sample. Recently, we have made even more concerted efforts to prevent late-hour esophagectomy at our institution. A prospective study of this subject may be unethical; therefore, we hope our present study attracts attention to enable the conduction of a multi-institutional study to obtain answers to any unanswered questions.

\section{Conclusions}

After-hours esophagectomy presents greater perioperative and long-term survival risks to patients with EC. We recommend that selective esophagectomy be canceled if the apparent start time is after 17:00. Further retrospective multi-institutional studies may be performed to corroborate our study's findings and explain how the late hours may affect prognosis.

\section{Acknowledgments}

Funding: None.

\section{Footnote}

Reporting Checklist: The authors have completed the STROBE reporting checklist. Available at http://dx.doi. org/10.21037/jtd-20-3141

Data Sharing Statement: Available at http://dx.doi. org/10.21037/jtd-20-3141

Conflicts of Interest: All authors have completed the ICMJE uniform disclosure form (available at http://dx.doi. org/10.21037/jtd-20-3141). The authors have no conflicts of interest to declare.

Ethical Statement: The authors are accountable for all aspects of this work in ensuring that questions related to the accuracy or integrity of any part of the work are appropriately investigated and resolved. The study was conducted in accordance with the Declaration of Helsinki (as revised in 2013). The study was approved by the institutional review board of Tongji Hospital, Tongji Medical College, Huazhong University of Science and Technology (NO.: TJ-C20201203) and individual consent for this retrospective analysis was waived.

Open Access Statement: This is an Open Access article distributed in accordance with the Creative Commons Attribution-NonCommercial-NoDerivs 4.0 International License (CC BY-NC-ND 4.0), which permits the noncommercial replication and distribution of the article with the strict proviso that no changes or edits are made and the original work is properly cited (including links to both the formal publication through the relevant DOI and the license). See: https://creativecommons.org/licenses/by-nc-nd/4.0/.

\section{References}

1. Kitagawa Y, Uno T, Oyama T, et al. Esophageal cancer practice guidelines 2017 edited by the Japan Esophageal Society: part 1. Esophagus 2019;16:1-24.

2. Kitagawa Y, Uno T, Oyama T, et al. Esophageal cancer practice guidelines 2017 edited by the Japan esophageal society: part 2. Esophagus 2019;16:25-43.

3. Ajani JA, D'Amico TA, Bentrem DJ, et al. Esophageal and Esophagogastric Junction Cancers, Version 2.2019, NCCN Clinical Practice Guidelines in Oncology. J Natl Compr Canc Netw 2019;17:855-83.

4. Birkmeyer JD, Siewers AE, Finlayson EVA, et al. Hospital volume and surgical mortality in the United States. N Engl J Med 2002;346:1128-37.

5. Finks JF, Osborne NH, Birkmeyer JD. Trends in hospital volume and operative mortality for high-risk surgery. $\mathrm{N}$ Engl J Med 2011;364:2128-37.

6. Sahni NR, Dalton M, Cutler DM, et al. Surgeon specialization and operative mortality in United States: retrospective analysis. BMJ 2016;354:i3571.

7. Mamidanna R, Ni Z, Anderson O, et al. Surgeon Volume and Cancer Esophagectomy, Gastrectomy, and Pancreatectomy: A Population-based Study in England. Ann Surg 2016;263:727-32.

8. Derogar M, Sadr-Azodi O, Johar A, et al. Hospital and surgeon volume in relation to survival after esophageal cancer surgery in a population-based study. J Clin Oncol 2013;31:551-7.

9. Brusselaers N, Mattsson F, Lagergren J. Hospital and 
surgeon volume in relation to long-term survival after oesophagectomy: systematic review and meta-analysis. Gut 2014;63:1393-400.

10. Markar SR, Mackenzie H, Lagergren P, et al. Surgeon Age in Relation to Prognosis After Esophageal Cancer Resection. Ann Surg 2018;268:100-5.

11. Gawande AA, Zinner MJ, Studdert DM et al. Analysis of errors reported by surgeons at three teaching hospitals. Surgery 2003;133:614-21.

12. Kelz RR, Tran TT, Hosokawa P, et al. Time-of-day effects on surgical outcomes in the private sector: a retrospective cohort study. J Am Coll Surg 2009;209:434-45.

13. Assali AR, Brosh D, Vaknin-Assa H, et al. The impact of circadian variation on outcomes in emergency acute anterior myocardial infarction percutaneous coronary intervention. Catheter Cardiovasc Interv 2006;67:221-6.

14. Kelz RR, Freeman KM, Hosokawa PW, et al. Time of day is associated with postoperative morbidity: an analysis of the national surgical quality improvement program data. Ann Surg 2008;247:544-52.

15. Ishiyama $Y$, Ishida F, Ooae S, et al. Surgical starting time in the morning versus the afternoon: propensity score matched analysis of operative outcomes following laparoscopic colectomy for colorectal cancer. Surg Endosc 2019;33:1769-76.

16. Badiyan SN, Ferraro DJ, Yaddanapudi S et al. Impact of time of day on outcomes after stereotactic radiosurgery for non-small cell lung cancer brain metastases. Cancer 2013;119:3563-9.

17. Kork F, Spies C, Conrad T, et al. Associations of postoperative mortality with the time of day, week and year. Anaesthesia 2018;73:711-8.

18. Lagergren J, Mattsson F, Lagergren P. Weekday of Esophageal Cancer Surgery and Its Relation to Prognosis. Ann Surg 2016;263:1133-7.

19. Charlson ME, Pompei P, Ales KL, et al. A new method of classifying prognostic comorbidity in longitudinal studies: development and validation. J Chronic Dis 1987;40:373-83.

20. Rice TW, Ishwaran H, Ferguson MK, et al. Cancer of the Esophagus and Esophagogastric Junction: An Eighth Edition Staging Primer. J Thorac Oncol 2017;12:36-42.

21. Society JE. Japanese Classification of Esophageal Cancer, 11th Edition: part I. Esophagus 2017;14:1-36.

22. Clavien PA, Barkun J, de Oliveira ML, et al. The ClavienDindo classification of surgical complications: five-year experience. Ann Surg 2009;250:187-96.

23. Katayama H, Kurokawa Y, Nakamura K, et al. Extended
Clavien-Dindo classification of surgical complications: Japan Clinical Oncology Group postoperative complications criteria. Surg Today 2016;46:668-85.

24. McMillan RR, Berger A, Sima CS, et al. Thirty-day mortality underestimates the risk of early death after major resections for thoracic malignancies. Ann Thorac Surg 2014;98:1769-74; discussion 1774-5.

25. Grigg OA, Farewell VT, Spiegelhalter DJ. Use of riskadjusted CUSUM and RSPRT charts for monitoring in medical contexts. Stat Methods Med Res 2003;12:147-70.

26. Linzey JR, Burke JF, Sabbagh MA, et al. The Effect of Surgical Start Time on Complications Associated With Neurological Surgeries. Neurosurgery 2018;83:501-7.

27. George TJ, Arnaoutakis GJ, Merlo CA, et al. Association of operative time of day with outcomes after thoracic organ transplant. JAMA 2011;305:2193-9.

28. Lohser J, Slinger P. Lung Injury After One-Lung Ventilation: A Review of the Pathophysiologic Mechanisms Affecting the Ventilated and the Collapsed Lung. Anesth Analg 2015;121:302-18.

29. Baba Y, Yoshida N, Shigaki H, et al. Prognostic Impact of Postoperative Complications in 502 Patients With Surgically Resected Esophageal Squamous Cell Carcinoma: A Retrospective Single-institutionStudy. Ann Surg 2016;264:305-11.

30. Hänecke K, Tiedemann S, Nachreiner F, et al. Accident risk as a function of hour at work and time of day as determined from accident data and exposure models for the German working population. Scand J Work Environ Health 1998;24 Suppl 3:43-8.

31. Lonze BE, Parsikia A, Feyssa EL, et al. Operative start times and complications after liver transplantation. Am J Transplant 2010;10:1842-9.

32. Peberdy MA, Ornato JP, Larkin GL, et al. Survival from in-hospital cardiac arrest during nights and weekends. JAMA 2008;299:785-92.

33. Wright MC, Phillips-Bute B, Mark JB, et al. Time of day effects on the incidence of anesthetic adverse events. Qual Saf Health Care 2006;15:258-63.

(English Language Editors: J. Jones and J. Chapnick)

Cite this article as: Tu DH, Qu R, Wang Q, Fu X. Afterhours esophagectomy may pose additional risk to patients with esophageal cancer. J Thorac Dis 2021;13(2):1118-1129. doi: $10.21037 /$ jtd-20-3141 